\title{
TEMA 7-2015: Micro ARN y Enfermedad pulmonar obstructiva crónica
}

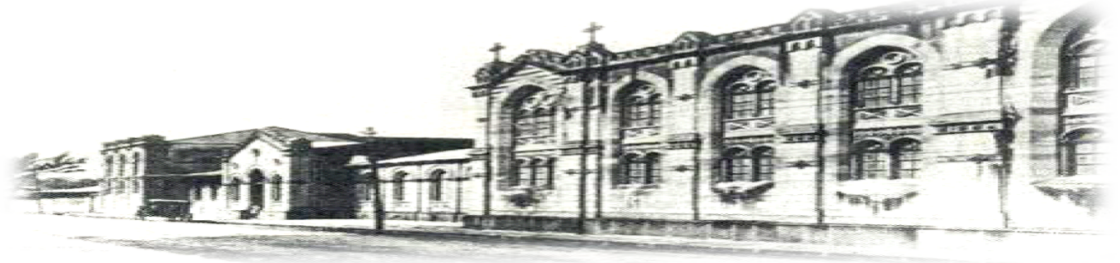

Hospital San quan de Dias. San José. Costa Rica. Fundado en 1845

Recibido: $\quad 08 / 03 / 2015$

Aceptado: $\quad 25 / 03 / 2015$

Ana Carolina Monge Zamora ${ }^{1}$ Carlos Fernando Estrada Garzona ${ }^{2}$

${ }^{1}$ Médica-Cirujana. Universidad de Costa Rica. Correo electrónico: ana c monge@hotmail.com

${ }^{2}$ Médico-Cirujano. Profesor del Departamento de Farmacología. Escuela de Medicina. Universidad de Costa Rica. Correo electrónico; drestradag@gmail.com

\section{KEY WORDS}

Pulmonary Embolism, risk factors, pathophysiology, diagnosis.

\section{INTRODUCCIÓN}

La enfermedad pulmonar obstructiva crónica (EPOC) es una enfermedad caracterizada por limitación irreversible, persistente y progresiva del flujo aéreo, asociada a una respuesta inflamatoria crónica en la vía aérea debido al depósito de partículas nocivas, principalmente relacionadas con el tabaquismo ${ }^{(1,2)}$.

Los mecanismos causantes de la enfermedad incluyen el daño celular, la inflamación y la reparación anormal de las células, causando destrucción enfisematosa del pulmón y enfermedad de vías aéreas inferiores ${ }^{(3,4)}$. Además de los factores mencionados, la susceptibilidad genética en cada paciente, incluyendo cambios epigenéticos, pueden jugar un papel importante en el inicio y progresión de la enfermedad ${ }^{(5)}$.

La Organización Mundial de la Salud (OMS) ha estimado que para el 2030 aumentarán en un $30 \%$ los casos diagnosticados de EPOC, siendo la quinta causa de discapacidad y la tercer causa de muerte a nivel mundial ${ }^{(5,6)}$. Actualmente, no existen tratamientos que puedan modificar el déficit progresivo de función pulmonar a largo plazo, siendo el transplante pulmonar la única intervención que puede mejorar significativamente la función pulmonar $\mathrm{y}$ disminuir la mortalidad en casos avanzados de $\operatorname{EPOC}^{(4,5)}$.

Dada la necesidad de evaluar nuevos posibles marcadores para diagnóstico temprano y nuevas opciones de tratamiento en esta patología, los microARN (miR) han surgido como una alternativa debido a su participación en mecanismos patológicos de la enfermedad pulmonar obstructiva crónica ${ }^{(7-10)}$. Esta revisión pretende abordar el concepto, mecanismo de 
acción y función de los microARN, así como su aplicación en el diagnóstico y tratamiento del paciente con EPOC.

\section{DISCUSIÓN}

\section{Definición: Micro ARN.}

Los micro ARN (miR) son una clase de pequeños ácidos ribonucleicos (ARN) no codificantes (18 a 25 nucléotidos), evolutivamente conservados, capaces de regular la expresión génica, dado que poseen función a nivel transcripcional y post-transcripcional, usualmente silenciando genes por medio de degradación de ARN mensajero $(\mathrm{ARNm})^{(8,11)}$.

Los miR fueron descubiertos inicialmente en 1993 en Caenorhabditis elegans y en Homo sapiens en el 2000, siendo involucrados en regulación postranscripcional específica para la expresión génica $^{(12)}$. En la actualidad, se ha documentado la existencia de cerca de $1.900 \mathrm{miR}$ en humanos, con distintas funciones fisiológicas y en la progresión, desarrollo o disminución de incidencia de diversas enfermedades ${ }^{(11)}$.

Los miR pueden localizarse en intrones o exones de genes codificantes de proteínas, o en sectores no codificantes del genoma. Para desarrollarse, primero ocurre transcripción por parte de la ARN polimerasa II o III, originando un $\mathrm{miR}$ primario (pri-miR), el cual posee forma de horquilla ${ }^{(11)}$. Posteriormente, por medio del complejo endonucleasa Drosha/DGCR8, se cortan ambos extremos del pri-miR, creando el precursor maduro del miR, llamado pre-miR, el cual contiene 70 a 100 nucleótidos. El pre-miR es exportado hacia el citoplasma por Exportin-5, donde de encuentra el complejo endonucleasa Dicer/TRBP, que lo divide en dos cadenas de forma helicoidal, de entre 19 y 25 nucleótidos.

Una de estas cadenas se convertirá en el miR maduro, mientras que la cadena análoga usualmente es degradada y aún no se le conoce una función específica ${ }^{(11,13)}$.

Se ha identificado que los miR se involucran en distintas etapas del desarrollo pulmonar. El cluster de miR-17 92, se expresa principalmente en células epiteliales progenitoras indiferenciadas y promueve su proliferación. El miR-127 y miR-351 se expresan transitoriamente en la fase sacular y alveolar, así como en células epiteliales pulmonares, lo que sugiere que forman parte de la reorganización $y$ diferenciación celular en el desarrollo pulmonar, mientras que miR-29a limita la proliferación en etapas tardías del desarrollo pulmonar ${ }^{(12)}$.

Los miR funcionan principalmente como supresores de expresión génica a nivel postranscripcional mediante la degradación del ARNm o inhibición de traducción protéica mediante la una unión en el extremo 3' de la región no codificante del ARNm, de manera que el grado de complementariedad va a determinar el mecanismo de supresión ${ }^{(13)}$. Cada miR es capaz de suprimir decenas o hasta centenas de genes, por lo que se encuentran involucrados en varias patologías. En ciertas condiciones pueden funcionar como oncogenes o supresores de tumores y su mutación o alteración puede asociarse al proceso de carcinogénesis ${ }^{(9,12)}$.

\section{Mecanismo de acción. Micro ARN (miR).}

Los miR son moduladores de la expresión de genes, que aumentan según el estímulo específico para cada uno ${ }^{(14)}$. Existen varios mecanismos de control de la función de los miR, siendo uno de ellos la inducción de la expresión de ciertos genes por factores de transcripción en respuesta a estímulos inflamatorios y estrés celular. Por otro lado, el mecanismo de modulación puede asociarse a la inhibición de la endonucleasa Dicer o modificaciones postranscripcionales. Por útlimo, se ha postulado que los miR son capaces de localizar gránulos de estrés, sin embargo aún se desconoce el mecanismo de esta forma de modulación. Además, los polimorfismos de nucleótido único (SNP) pueden afectar y causar un cambio de function del miR, siendo este otro mecanismo de acción $^{(15)}$.

Los miR son estables en sangre periférica debido a su resistencia a las ARNasas endógenas ${ }^{(16)}$. Se ha evidenciado que los miR pueden expresarse de forma específica, ya sea por su presencia en un tejido o por aumento de su expresión en sangre periférica $u$ otros fluidos corporales. De esta manera, los miR son secretados como microvesiculas hacia la circulación y por su gran estabilidad en tejidos y fluidos corporales, tienen potencial importante como marcadores diagnósticos $^{(17)}$. 


\section{Rol de los micro ARN en Inflamación.}

Los miR se encuentran involucrados en varios procesos físiológicos y patológicos asociados al proceso de inflamación, un fenómeno fisiopatológico del paciente con $\operatorname{EPOC}^{(18,19)}$. Los micro RNA regulan la diferenciación de células inflamatorias $\mathrm{y}$ tienen una función como reguladores de la respuesta immune ${ }^{(20,21)}$. Se ha documentado que los miR se involucran en el desarrollo de linfocitos $\mathrm{T}$ y $\mathrm{B}$. El miR-150 bloquea la producción de células $\mathrm{B}$ maduras por desregulación del factor de transcripción. Por otro lado, la sobreexpresión de miR-181 aumenta de forma importante la cantidad de células B, así como aumenta la sensibilidad de receptores de antígenos de las células $\mathrm{T}^{(15)}$.

En la vía aérea, la inmunidad innata está dada por macrófagos alveolares, neutrófilos, células epiteliales y los receptores Toll-like (TLR) que detectan antígenos microbianos y son los encargados de iniciar cascadas de señales que culminan en expresión de genes de inflamación ${ }^{(15)}$.

Los TLRs pueden inducir la expresión de miR, para activar la expresión de genes inflamatorios que producen posteriormente citoquinas, tales como interleucinas (IL) y Factor de Necrosis Tumoral alfa (TNF- $\alpha$ ) en células de inmunidad innata, como los macrófagos. De esta manera, los lipopolisacaridos, antígenos microbianos y ligando principal para TLR4, se han asociado con inducción de miR-155, miR-146, miR-9 y $\operatorname{miR}-21^{(15,18-21)}$.

\section{Micro ARN y Tabaquismo}

El depósito de partículas de tabaco en las vías aéreas inferiores en el paciente con EPOC, desencadena un proceso inflamatorio crónico que evoluciona hacia daño pulmonar irreversible, causando enfisema como complicación ${ }^{(4,18-26)}$. El tabaquismo aumenta la expresión de 175 genes que causan estos cambios y la susceptibilidad genética presente en los fumadores define el inicio y progresión de la enfermedad ${ }^{(14,22-25)}$. El miR-218 y miR-128 disminuyen su expresión en el paciente expuesto a fumado, mientras que miR-31 aumenta en las células de la vía aérea de fumadores. Además, el miR let-7c disminuye su expresión en aquellos pacientes fumadores que padecen de EPOC $^{(19)}$.
Otro marcador interesante en el paciente con EPOC es el miR-126, el cual disminuye hasta dos veces de su valor normal por exposición al humo, por lo que permite relacionar la noxa causada por exposición a partículas de tabaco en vía aérea inferior y la predisposición a enfisema y $\operatorname{EPOC}^{(22)}$.

El miR-218 es un marcador útil para diferenciar entre células epiteliales bronquiales de fumadores en contraste con no fumadores ${ }^{(15)}$. Además, el miR-452 también se ha asociado al aumento de expresión de la metaloproteinasa de matriz 12 (MMP-12) implicada en el desarrollo de enfisema ${ }^{(12)}$.

La persistencia de las alteraciones en la expresión de los miR a nivel pulmonar son proporcionales a la duración de la exposición al humo del tabaco ${ }^{(26)}$. De esta manera, el miR-146a se expresa en fumadores y se asocia a una producción elevada de prostaglandina E2 (PGE2), un mediador lipídico que causa un aumento en la cascada inflamatoria y progresión hacia lesión pulmonar $^{(23)}$. Por otro lado, el let-7c se expresa en células bronquiales, especialmente en macrófagos, causando un aumento en la inflamación y progresión de $\operatorname{EPOC}^{(19,27)}$.

\section{Micro ARN y EPOC}

El paciente con EPOC presenta un estado de inflamación sistémica, siendo la apoptosis el principal mecanismo de destrucción alveolar ${ }^{(28)}$.

Otro de los factores implicados en la fisiopatología de esta enfermedad es el estrés oxidativo, el cual activa otras vías intracelulares que median en la inflamación ${ }^{(28,29)}$. Sin embargo, los factores de transcripción y eventos epigenéticos variables entre cada paciente son importantes para definir la modulación de la respuesta inflamatoria ${ }^{(16)}$.

La destrucción del tejido pulmonar en pacientes con EPOC y enfisema no se describe únicamente por mecanismos inflamatorios, sino que también mecanismos no inflamatorios, como la disminución de la expresión de la proteína del factor inducido por hipoxia-alfa1 (HIF-1alfa) que varía según la disfunción pulmonar ${ }^{(30,31)}$. Sin embargo, cabe señalar que los principales miR presentes en EPOC van a estar asociados a genes que regulan la inflamación, ya que es su mecanismo fisiopatológico principal ${ }^{(17,32)}$. En 
este sentido, la dismunición de let-7c y miR$125 \mathrm{~b}$, afecta genes como el del receptor de TNF tipo $2^{(14,30)}$. De igual manera, la reducción de la expresión del miR-146a implica un aumento en la reacción inflamatoria dado una menor degradación de la COX-2 $2^{(23)}$, mientras que un aumento en la expresión de miR-34a y miR$199 a-5 p$ en pacientes con EPOC y enfisema se asocia a disminución de la función pulmonar ${ }^{(30)}$.

Se ha documentado disminución en la expresión de miR-1, miR-133 y miR-206, principalmente en el diafragma de pacientes con EPOC, atribuido al aumento de carga inspiratoria en el músculo de estos pacientes ${ }^{(34,35)}$.

La expresión de miR varía en tejido pulmonar sano y en los tejidos enfermos o con exposición al tabaco, un concepto que permite utilizar la identificación de los microARN como biomarcadores en EPOC (Tabla 1) ${ }^{(33)}$. Además, algunos miR como el miR-34a y miR-199a-5p se relacionan a la severidad de la enfermedad pulmonar y no con el fumado o el cáncer pulmonar, considerado otro factor que puede afectar la expresión de miR, por lo que surgen como posibles marcadores diagnósticos para EPOC $^{(36,37)}$.

La expresión de proteína p53, importante en patogenia de EPOC, se relaciona directamente con miR-34a, mientras que la expresión de la proteína HIF-1alfa se relaciona inversamente con la expresión de miR-199a-5 $\mathrm{p}^{(30)}$. La identificación del miR-362-3p y miR-338-3p se considera un potencial marcador importante para diagnóstico de EPOC dado permite diferenciar esta enfermedad de otra patología con componente inflamatorio como el asma bronquial $^{(17)}$.

Tabla 1. Expresión de micro ARN (miR) asociados a Enfermedad pulmonar obstructiva crónica (EPOC) 8,12

\begin{tabular}{ll|}
\hline Expresión aumentada & Expresión disminuida \\
\hline miR-132 & miR-29b \\
miR-145 & miR-34a \\
miR-212 & miR-98 \\
miR-223 & miR-146b-5p \\
miR-342-5p & miR-193a-5p \\
miR-422a & miR-218 \\
miR-423-5p & miR-324-5p \\
miR-1274 & miR-923 \\
miR-101 & miR-937 \\
miR-144 & miR-125b-1 \\
miR-146a & miR-452 \\
\hline
\end{tabular}

Con el descubrimiento de la función de los miR en la fisiopatología de EPOC, la alteración o modulación de estos RNA se convierte en una interesante meta terapéutica ${ }^{(38,39)}$. La inhibición del miR-145, involucrado en la respuesta de reclutamiento de eosinófilos, disminuye la producción de citoquinas IL-13 e IL-5, asociando un alivio importante en la sintomatología inflamatoria en asma bronquial ${ }^{(17)}$. Sin embargo, la modulación de los miR en EPOC no cuenta aún con estudios clínicos que demuestren la efectividad de esta intervención ${ }^{(15)}$.

Cabe señalar que en las patologías pulmonares es frecuente el traslape de función de miR entre las distintas enfermedades dado que poseen efecto sobre varios genes ${ }^{(40)}$. El miR-221, cuya función implica el control de los inhibidores de ciclina en las células de músculo liso de vía aérea, pueden identificarse tanto en pacientes con asma bronquial como con cualquier otra patología respiratoria que afecte dichas células ${ }^{(16)}$. Muchos de los miR inflamatorios, como el miR-146, miR-342 y miR-142-3p se encuentran presentes en asma bronquial y en EPOC, un hallazgo que demuestra el posible traslape de miR en enfermedades pulmonares según la similitud de mecanismos fisiopatológicos. ${ }^{(41)}$

\section{CONCLUSIONES}

Los microARN (miR) son una clase de pequeños ácidos ribonucleicos (ARN) no codificantes, con la capacidad de regular la expresión génica, cuya identificación podría orientar el diagnóstico y plantear nuevos objetivos terapéuticos en el paciente con EPOC.

\section{REFERENCIAS BIBLIOGRÁFICAS}

1. Barreiro E Gea J. Epigenetics and muscle dysfunction in chronic obstructive pulmonary disease. Translational Research. 2014;165(1):61-73.

2. Grundy S Plumb J Lea et al. Down Regulation of $T$ Cell Receptor Expression in COPD Pulmonary CD8 Cells. PLoS ONE. 2013;8(8):1-11.

3. Ezzie ME Crawford M Cho J. et al. Gene expression networks in COPD: microRNA and $m R N A$ regulation. Thorax. 2012;67(2):122-131. 
4. Christenson S Brandsma CA Campbell JD et al. miR-638 regulates gene expression networks associated with emphysematous lung destruction. Genome Medicine. 2013;5(12):1-13.

5. Schamberger AC Mise N Meiners S Eickelberg O. Epigenetic mecanisms in COPD: implications for pathogenesis and drug discovery. Expert Opinion Drug Discovery. 2014;9(6):609-628.

6. Provinciali M Cardelli M Marchegiani F. Inflammation, chronic obstructive pulmonary disease and aging. Current Opinion in Pulmonary Medicine. 2011; 17(1):S3-S10.

7. Li LJ Gao LB Lv ML et al. Association between SNPs in pre-miRNA and risk of chronic obstructive pulmonary disease. Clinical Biochemistry. 2011;44(10-11): 813-816.

8. Molina-Pinelo S Pastor MD Suarez R et al. MicroRNA clusters: dysregulation in lung adenocarcinoma and COPD. European Respiratory Journal. 2014;43(6): 1740-1749.

9. Pagdin PL. MicroRNAs in lung diseases. Thorax. 2011;67(2):183-184.

10. Chavali S Bruhn S Tiemann et al. MicroRNAs act complementarily to regulate disease-related $m R N A$ modules in human diseases. RNA Journal, 2013;19 (11):1552-1562.

11. Angulo M Lecuona E Iasha Sznajder J. Rol de los microARN en las enfermedades pulmonares. Archivos de Bronconeumologia. 2012;48(9):325-330.

12. Sessa R Hata A. Role of microRNAs in lung development and pulmonary diseases. Pulmonary Circulation. 2013;3 (2):315-328.

13. Barreiro E Sznajder JI. Epigenetic regulation of muscle phenotype and adaptation: a potential role in COPD muscle dysfunction. Journal of Applied Physiology. 2013;114(9):1263-1272.

14. Adcock IM Caramori G Barnes PJ. Chronic Obstructive Pulmonary Disease and Lung Cancer: New Molecular Insights. Respiration. 2011;81(4):265284.

15. Ogelsby I McElvaney N Greene C. MicroRNAs in inflammatory lung disease master regulators or target practice? Respiratory Research. 2010;11:1-13.
16. Donaldson A Natanek SA Lewis A et al. Increased skeletal muscle-specific microRNA in the blood of patients with COPD. Thorax. 2013;68(12):11401149.

17. Wang M Huang Y Liang Z et al. Plasma miRNAs might be promising biomarkers of chronic obstructive pulmonary disease. The Clinical Respiratory Journal. 2014: doi: 10.1111/crj.12194.

18. Basma H Gunji Y Iwasawa et al. Reprogramming of COPD lung fibroblasts through formation of induced pluripotent stem cells. American Journal of Physiology and Lung Cell Molecular Physiology. 2014;306(6):L552-L565.

19. Brusselle GG Jaos GF Bracke KR. New insights into the immunology of chronic obstructive pulmonary disease. Lancet. 2011;378(9795):1015-1026.

20. Kishore A Borucka J Petrkova J Petrek M. Novel insights into miRNA in Lung and Heart Inflammatory Diseases. Mediators of Inflammation. 2014: doi: 10.1155/2014/259131.

21. Plank M Maltby S Mattes J Foster P. Targeting translational control as a novel way to treat inflammatory disease: the emerging role of MicroRNAs. Clinical and Experimental Allergy. 2013;43(9):981-999.

22. Francis SM Davidson MR Tan ME et al. MicroRNA-34c is associated with emphysema severity and modulates SERPINE1 expression. BMC Genomics. 2014;15(88):1-8.

23. Sato T Liu X Nelson A et al. Reduced miR-146a Increases Prostaglandin E2 in Chronic Obstructive Pulmonary Disease Fibroblasts. American Journal of Respiration and Critical Care Medicine. 2010;182(8):1020-1029.

24. Hassan T Carroll TP Buckley PG et al. miR-199-5p Silencing Regulates the Unfolded Protein Response in Chronic Obstructive Pulmonary Disease and AlfalAntitrypsin Deficiency. American Journal of Respiration and Critical Care Medicine. 2014;189(3):263-273.

25. Mizuno $S$ Yasuo $M$ Bogaard $H$ Kraskauskas D Natarajan R Voelkel N. Inhibition of histone deacetylase causes emphysema. American Journal of Physiology and Lung Cell Molecular Physiology. 2010;300(3):L402-L413. 
26. Takahashi K Yokota S Tatsumi N Fukami T Yokoi T Nakajima M. Cigarette smoking substantially alters plasma microRNA profiles in healthy subjects. Toxicology and Applied Pharmacology. 2013;272(1):154-160.

27. Gross TJ Powers LS Boudreau RL et al. A microRNA-processing defect in smokers' macrophages is linked to SUMOylation of the endonuclease DICER. Journal of Biological Chemistry. 2014; 289(18):12823-12834.

28. Yang IA Rela V Wright CM et al. Common pathogenic mechanisms and pathways in the development of COPD and lung cancer. Expert Opinion Therapy Targets. 2011;15(4):439-456.

29. Bowman RV Wright CM Davidson MR Francis SM Yang IA Fong KM. Epigenomic targets for the treatment of respiratory disease. Expert Opinion Therapy Targets. 2009;13(6):625-640.

30. Mizuno S Bogaard HJ Gomez-Arroyo J et al. MicroRNA-199a-5p Is Associated with Hypoxia-Inducible Factor-1alfa Expression in Lungs From Patients with COPD. Chest. 2012;142(3):663-672.

31. Yue J Guan J Wang X et al. MicroRNA206 is involved in hypoxia-induced pulmonary hypertension through targeting of the HIF-1alfa/Fhl-1 pathway. Laboratory Investigation. 2013;93(7):748759.

32. Akbas F Coskunpinar E Aynaci E Musteri Oltulu Y Yildiz P. Analysis of serum micro-RNAs as potential biomarker in Chronic Obstructive Pulmonary Disease. Experimental Lung Research. 2012;38(6):1-9.

33. Banerjee A Luettich K. MicroRNAs as potential biomarkers of smoking-related diseases. Biomarkers Medicine. 2012;6 (5):671-684.

34. Puig-Vilanova E Aguilo R RodriguezFuster A Martinez-Llorens J Gea J Barreiro E. Epigenetic Mechanisms in Respiratory Muscle Dysfunction of Patients with Chronic Obstructive Pulmonary Disease. PLoS ONE. 2014;9(11): e111514.

doi: 10.1371/journal.pone.0111514

35. Donaldson A Natanek SA Lewis A et al. Increased skeletal muscle-specific microRNA in the blood of patients with
COPD. Thorax. 2013;68(12):11401149.

36. Leidinger $\mathrm{P}$ Keller A Borries A et al. Specific peripheral miRNA profiles for distinguishing lung cancer from COPD. Lung Cancer. 2011;74(1):41-47.

37. Lewis A Riddoch-Contreras J Natanek $\mathrm{S}$ et al. Downregulation of the serum response factor/miR-1 axis in the quadriceps of patients with COPD. Thorax. 2012;67(1):26-34.

38. Novak J Kruzliak P Bienertova-Vasku J Slaby O Novak M. MicroRNA-206: a Promising Theranostic Marker. Theranostics. 2014;4(2):119-133.

39. Ru Y Kechris K Tabakoff B et al. The multiMiR $R$ package and database: integration of microRNA-target interactions along with their disease and drug associations. Nucleic Acids Research. 2014;42(17):1-10.

40. Pinkerton M Chinchilli V Banta E et al. Differential expression of microRNAs in exhaled breath condensates of patients with asthma, patients with chronic obstructive pulmonary disease, and healthy adults. Journal of Allergy and Clinical Inmunology. 2013;132(1):217219.

41. Perry MP Tsitsiou E Austin PJ et al. Role of non-coding RNAs in maintaining primary airway smooth muscle cells. Respiratory Research. 2014;15 (58):1-12.

\section{DECLARACIÓN DE CONFLICTO DE IN- TERESES}

Los autores declaran que no existen conflictos de interés. 